$2008 / 07 / 10$

\title{
Hook lengths and shifted parts of partitions
}

\author{
Guo-Niu HAN
}

\begin{abstract}
Some conjectures on partition hook lengths, recently stated by the author, have been proved and generalized by Stanley, who also needed a formula by Andrews, Goulden and Jackson on symmetric functions to complete his derivation. Another identity on symmetric functions can be used instead. The purpose of this note is to prove it.
\end{abstract}

\section{Introduction}

The hook lengths of partitions are widely studied in the Theory of Partitions, in Algebraic Combinatorics and Group Representation Theory. The basic notions needed here can be found in [St99, p.287; La01, p.1]. A partition $\lambda$ is a sequence of positive integers $\lambda=\left(\lambda_{1}, \lambda_{2}, \cdots, \lambda_{\ell}\right)$ such that $\lambda_{1} \geq \lambda_{2} \geq \cdots \geq \lambda_{\ell}>0$. The integers $\left(\lambda_{i}\right)_{i=1,2, \ldots, \ell}$ are called the parts of $\lambda$, the number $\ell$ of parts being the length of $\lambda$ denoted by $\ell(\lambda)$. The sum of its parts $\lambda_{1}+\lambda_{2}+\cdots+\lambda_{\ell}$ is denoted by $|\lambda|$. Let $n$ be an integer, a partition $\lambda$ is said to be a partition of $n$ if $|\lambda|=n$. We write $\lambda \vdash n$. Each partition can be represented by its Ferrers diagram. For each box $v$ in the Ferrers diagram of a partition $\lambda$, or for each box $v$ in $\lambda$, for short, define the hook length of $v$, denoted by $h_{v}(\lambda)$ or $h_{v}$, to be the number of boxes $u$ such that $u=v$, or $u$ lies in the same column as $v$ and above $v$, or in the same row as $v$ and to the right of $v$. The product of all hook lengths of $\lambda$ is denoted by $H_{\lambda}$.

The hook length plays an important role in Algebraic Combinatorics thanks to the famous hook formula due to Frame, Robinson and Thrall [FRT54]

$$
f_{\lambda}=\frac{n !}{H_{\lambda}},
$$

where $f_{\lambda}$ is the number of standard Young tableaux of shape $\lambda$.

For each partition $\lambda$ let $\lambda \backslash 1$ be the set of all partitions $\mu$ obtained from $\lambda$ by erasing one corner of $\lambda$. By the very construction of the standard Young tableaux and (1.1) we have

$$
f_{\lambda}=\sum_{\mu \in \lambda \backslash 1} f_{\mu}
$$

and then

$$
\frac{n}{H_{\lambda}}=\sum_{\mu \in \lambda \backslash 1} \frac{1}{H_{\mu}} .
$$


In this note we establish the following perturbation of formula (1.3). Define the $g$-function of a partition $\lambda$ of $n$ to be

$$
g_{\lambda}(x)=\prod_{i=1}^{n}\left(x+\lambda_{i}-i\right)
$$

where $\lambda_{i}=0$ for $i \geq \ell(\lambda)+1$.

Theorem 1.1. Let $x$ be a formal parameter. For each partition $\lambda$ we have

$$
\frac{g_{\lambda}(x+1)-g_{\lambda}(x)}{H_{\lambda}}=\sum_{\mu \in \lambda \backslash 1} \frac{g_{\mu}(x)}{H_{\mu}} .
$$

Theorem 1.1 is proved in Section 2. Some equivalent forms of Theorem 1.1 and remarks are given in Section 4. As an application we prove (see Section 3) the following result due to Stanley [St08].

Theorem 1.2. Let $p, e$ and $s$ be the usual symmetric functions [Ma95, Chap.I]. Then

$$
\sum_{k=0}^{n}\left(\begin{array}{c}
x+k-1 \\
k
\end{array}\right) p_{1}^{k} e_{n-k}=\sum_{\lambda \vdash n} H_{\lambda}^{-1} g_{\lambda}(x+n) s_{\lambda} .
$$

Recently, the author stated some conjectures on partition hook lengths [Ha08a], which were suggested by hook length expansion techniques (see [Ha08b]). Later, Conjecture 3.1 in [Ha08a] was proved by Stanley [St08]. One step of his proof is formula (1.6), based on a result by Andrews, Goulden and Jackson [AGJ88]. In this paper we provide a simple and direct proof of formula (1.6).

Remark. Let $D$ be the difference operator defined by

$$
D(f(x))=f(x+1)-f(x) .
$$

By iterating formula (1.5) we obtain

$$
D^{n} \frac{g_{\lambda}(x)}{H_{\lambda}}=f_{\lambda},
$$

which is precisely the hook length formula (1.1). 


\section{Proof of Theorem 1.1}

Let

$$
\epsilon(x)=\frac{g_{\lambda}(x+1)-g_{\lambda}(x)}{H_{\lambda}}-\sum_{\mu \in \lambda \backslash 1} \frac{g_{\mu}(x)}{H_{\mu}} .
$$

We see that $\epsilon(x)$ is a polynomial in $x$ whose degree is less than or equal to $n$. Moreover

$$
\left[x^{n}\right] \epsilon(x)=\left[x^{n}\right] \frac{g_{\lambda}(x+1)-g_{\lambda}(x)}{H_{\lambda}}=0 .
$$

Furthermore,

$$
\left[x^{n-1}\right] g_{\lambda}(x+1)=\sum_{i=1}^{n}\left(\lambda_{i}-i+1\right)=n+\sum_{i=1}^{n}\left(\lambda_{i}-i\right)=n+\left[x^{n-1}\right] g_{\lambda}(x)
$$

and

$$
\left[x^{n-1}\right] \epsilon(x)=\left[x^{n-1}\right] \frac{g_{\lambda}(x+1)-g_{\lambda}(x)}{H_{\lambda}}-\sum_{\mu \in \lambda \backslash 1} \frac{1}{H_{\mu}}=\frac{n}{H_{\lambda}}-\sum_{\mu \in \lambda \backslash 1} \frac{1}{H_{\mu}}=0 .
$$

The last equality is guaranteed by (1.3), so that $\epsilon(x)$ is a polynomial in $x$ whose degree is less than and equal to $n-2$. To prove that $\epsilon(x)$ is actually zero, it suffices to find $n-1$ distinct values for $x$ such that $\epsilon(x)=0$. In the following we prove that $\epsilon\left(i-\lambda_{i}\right)=0$ for $i-\lambda_{i}$ for $i=1,2, \ldots, n-1$.

If $\lambda_{i}=\lambda_{i+1}$, or if the $i$-th row has no corner, the factor $x+\lambda_{i}-i$ lies in $g_{\lambda}(x)$ and also in $g_{\mu}(x)$ for all $\mu \in \lambda \backslash 1$. The factor $(x+1)+\lambda_{i+1}-(i+1)=$ $x+\lambda_{i}-i$ is furthermore in $g_{\lambda}(x+1)$, so that $\epsilon\left(i-\lambda_{i}\right)=0$.

Next, if $\lambda_{i} \geq \lambda_{i+1}+1$, or if the $i$-th row has a corner, the factor $x+\lambda_{i}-i$ lies in $g_{\lambda}(x)$ and $g_{\mu}(x)$ for all $\mu \in \lambda \backslash 1$, except for $\mu=\lambda^{\prime}$, which is the partition obtained from $\lambda$ by erasing the corner from the $i$-th row. In this case equality (2.1) becomes

$$
\epsilon\left(i-\lambda_{i}\right)=\frac{g_{\lambda}\left(i-\lambda_{i}+1\right)}{H_{\lambda}}-\frac{g_{\lambda^{\prime}}\left(i-\lambda_{i}\right)}{H_{\lambda^{\prime}}}
$$

For proving Theorem 1.1, it remains to prove $\epsilon\left(i-\lambda_{i}\right)=0$ or

$$
\frac{H_{\lambda}}{H_{\lambda^{\prime}}}=\frac{g_{\lambda}\left(i-\lambda_{i}+1\right)}{g_{\lambda^{\prime}}\left(i-\lambda_{i}\right)}
$$


Consider the following product

$$
\frac{g_{\lambda}(x+1)}{g_{\lambda^{\prime}}(x)}=\frac{\prod_{j=1}^{n}\left(x+\lambda_{j}-j+1\right)}{\prod_{j=1}^{n-1}\left(x+\lambda_{j}^{\prime}-j\right)} .
$$

The set of all $1 \leq j \leq n-1$ such that $\lambda_{j}>\lambda_{j+1}$ is denoted by $\mathcal{T}$. For $1 \leq j \leq n-1$ and $j \notin \mathcal{T}$ (which implies that $j \neq i$ and $\lambda_{j}^{\prime}=\lambda_{j}=\lambda_{j+1}$ ), the numerator contains $x+\lambda_{j+1}-(j+1)+1=x+\lambda_{j}-j$ and the denominator also contains $x+\lambda_{j}^{\prime}-j=x+\lambda_{j}-j$. After cancellation of those common factors, (2.3) becomes

$$
\frac{g_{\lambda}(x+1)}{g_{\lambda^{\prime}}(x)}=\frac{\prod_{j \in \mathcal{B}}\left(x+\lambda_{j}-j+1\right)}{\prod_{j \in \mathcal{T}}\left(x+\lambda_{j}^{\prime}-j\right)}
$$

where $\mathcal{B}=\{1\} \cup\{i+1 \mid i \in \mathcal{T}\}$. Letting $x=i-\lambda_{i}$ in (2.4) yields

$$
\frac{g_{\lambda}\left(i-\lambda_{i}+1\right)}{g_{\lambda^{\prime}}\left(i-\lambda_{i}\right)}=\frac{\prod_{j \in \mathcal{B}}\left(i-\lambda_{i}+\lambda_{j}-j+1\right)}{\prod_{j \in \mathcal{T}}\left(i-\lambda_{i}+\lambda_{j}^{\prime}-j\right)} .
$$

We distinguish the factors in the right-hand side of (2.5) as follows.

(C1) For $j \in \mathcal{B}$ and $j>i, i-\lambda_{i}+\lambda_{j}-j+1=-\left(\lambda_{i}-\lambda_{j}+j-i-1\right)=$ $-h_{v}(\lambda)$, where $v$ is the box $\left(i, \lambda_{j}+1\right)$ in $\lambda$.

(C2) For $j \in \mathcal{B}$ and $j \leq i, i-\lambda_{i}+\lambda_{j}-j+1=h_{v}(\lambda)$, where $v$ is the $\operatorname{box}\left(j, \lambda_{i}\right)$ in $\lambda$.

(C3) For $j \in \mathcal{T}$ and $j>i, i-\lambda_{i}+\lambda_{j}-j=-\left(\lambda_{i}-\lambda_{j}+j-i\right)=-h_{u}\left(\lambda^{\prime}\right)$, where $u$ is the box $\left(i, \lambda_{j}\right)$ in $\lambda^{\prime}$.

(C4) For $j \in \mathcal{T}$ and $j<i, i-\lambda_{i}+\lambda_{j}-j=h_{u}\left(\lambda^{\prime}\right)$, where $u$ is the box $\left(j, \lambda_{i}\right)$ in $\lambda^{\prime}$.

(C5) For $j \in \mathcal{T}$ and $j=i, i-\lambda_{i}+\lambda_{j}^{\prime}-j=i-\lambda_{i}+\lambda_{i}^{\prime}-i=-1$. See Fig. 2.3 and 2.4 for an example.

Since each $j \in \mathcal{B}$ such that $j>i$ is associated with $j-1 \in \mathcal{T}$ and $j-1 \geq i$, the right-hand side of $(2.5)$ is positive and can be re-written

$$
\frac{g_{\lambda}\left(i-\lambda_{i}+1\right)}{g_{\lambda^{\prime}}\left(i-\lambda_{i}\right)}=\frac{\prod_{v} h_{v}(\lambda)}{\prod_{u} h_{u}\left(\lambda^{\prime}\right)}
$$

where $v, u$ range over the boxes described in (C1)-(C4). Finally $H_{\lambda} / H_{\lambda^{\prime}}$ is equal to the right-hand side of (2.6), since the hook lengths of all other boxes cancel. We have completed the proof of $(2.2)$. 
For example, consider the partition $\lambda=55331$ and $i=4$. We have $\lambda^{\prime}=55321$ and

$$
\frac{H_{\lambda}}{H_{\lambda^{\prime}}}=\frac{4 \cdot 2 \cdot 1 \cdot 2 \cdot 5 \cdot 6}{3 \cdot 1 \cdot 1 \cdot 4 \cdot 5} .=\frac{4 \cdot 2 \cdot 2 \cdot 6}{3 \cdot 4} .
$$

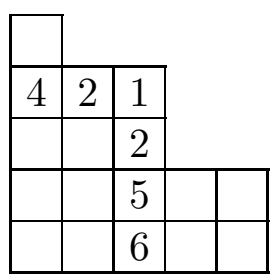

Fig. 2.1. Hook lengths of $\lambda$

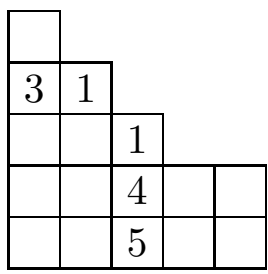

Fig. 2.2. Hook lengths of $\lambda^{\prime}$

On the other hand, $\mathcal{T}=\{2,4,5\}, \mathcal{B}=\{1,3,5,6\}$ and

$$
\frac{g_{\lambda}(x+1)}{g_{\lambda^{\prime}}(x)}=\frac{(x+5)(x+1)(x-3)(x-5)}{(x+3)(x-2)(x-4)} .
$$

Letting $x=i-\lambda_{i}=4-3=1$ yields

$$
\frac{g_{\lambda}(2)}{g_{\lambda^{\prime}}(1)}=\frac{(6)(2)(-2)(-4)}{(4)(-1)(-3)}=\frac{6 \cdot 2 \cdot 2 \cdot 4}{4 \cdot 3} \text {. }
$$

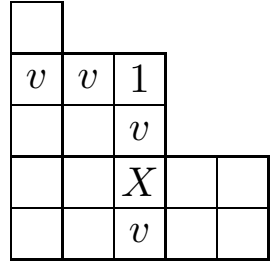

Fig. 2.3. The boxes $v$ in $\lambda$

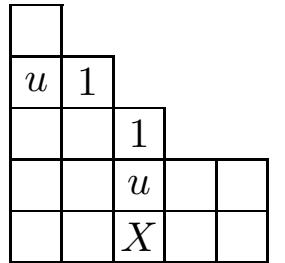

Fig. 2.4. The boxes $u$ in $\lambda^{\prime}$

\section{Proof of Theorem 1.2}

Let $R_{n}(x)$ be the right-hand side of (1.6). By Theorem 1.1

$$
\begin{aligned}
R_{n}(x) & =\sum_{\lambda \vdash n}\left(\frac{g_{\lambda}(x+n-1)}{H_{\lambda}}+\sum_{\mu \in \lambda \backslash 1} \frac{g_{\mu}(x+n-1)}{H_{\mu}}\right) s_{\lambda} \\
& =R_{n}(x-1)+\sum_{\lambda \vdash n} \sum_{\mu \in \lambda \backslash 1} \frac{g_{\mu}(x+n-1)}{H_{\mu}} s_{\lambda} \\
& =R_{n}(x-1)+\sum_{\mu \vdash n-1} \sum_{\lambda: \mu \in \lambda \backslash 1} \frac{g_{\mu}(x+n-1)}{H_{\mu}} s_{\lambda} \\
& =R_{n}(x-1)+\sum_{\mu \vdash n-1} \frac{g_{\mu}(x+n-1)}{H_{\mu}} p_{1} s_{\mu},
\end{aligned}
$$


where the next to last equality is

$$
\sum_{\lambda: \mu \in \lambda \backslash 1} s_{\lambda}=p_{1} s_{\mu}
$$

by using Pieri's rule [Ma95, p.73]. We obtain the following recurrence for $R_{n}(x)$.

$$
R_{n}(x)=R_{n}(x-1)+p_{1} R_{n-1}(x)
$$

Let $L_{n}(x)$ be the left-hand side of (1.6). Using elementary properties of binomial coefficients

$$
\begin{aligned}
L_{n}(x) & =\sum_{k=0}^{n}\left(\begin{array}{c}
x+k-1 \\
k
\end{array}\right) p_{1}^{k} e_{n-k} \\
& =e_{n}+\sum_{k=1}^{n}\left(\left(\begin{array}{c}
x+k-2 \\
k
\end{array}\right)+\left(\begin{array}{c}
x+k-2 \\
k-1
\end{array}\right)\right) p_{1}^{k} e_{n-k} \\
& =L_{n}(x-1)+p_{1} \sum_{k=1}^{n}\left(\begin{array}{c}
x+k-2 \\
k-1
\end{array}\right) p_{1}^{k-1} e_{n-k} \\
& =L_{n}(x-1)+p_{1} L_{n-1}(x) .
\end{aligned}
$$

We verify that $L_{1}(x)=R_{1}(x)$ and $L_{n}(0)=R_{n}(0)$, so that $L_{n}(x)=R_{n}(x)$ by (3.1) and (3.2).

\section{Equivalent forms and further remarks}

Let $\lambda=\lambda_{1} \lambda_{2} \cdots \lambda_{\ell}$ be a partition of $n$. The set of all $1 \leq j \leq n$ such that $\lambda_{j}>\lambda_{j+1}$ is denoted by $\mathcal{T}$ and let $\mathcal{B}=\{1\} \cup\{i+1 \mid i \in \mathcal{T}\}$. Those two sets can be viewed as the in-corner and out-corner index sets, respectively. Notice that $\# \mathcal{B}=\# \mathcal{T}+1$. For each $i \in \mathcal{T}$ we define $\lambda^{i-}$ to be the partition of $n-1$ obtained form $\lambda$ by erasing the right-most box from the $i$-th row. Hence

$$
\lambda \backslash 1=\left\{\lambda^{i-} \mid i \in \mathcal{T}\right\}
$$

We verify that

$$
g_{\lambda^{i-}}(x)=\frac{g_{\lambda}(x)\left(x+\lambda_{i}-i-1\right)}{\left(x+\lambda_{i}-i\right)(x-n)} .
$$

From Theorem 1.1

$$
\frac{g_{\lambda}(x+1)-g_{\lambda}(x)}{H_{\lambda}}=\sum_{i \in \mathcal{T}} \frac{g_{\lambda}(x)\left(x+\lambda_{i}-i-1\right)}{\left(x+\lambda_{i}-i\right)(x-n)} \frac{1}{H_{\lambda}^{i-}}
$$


or

$$
\sum_{i \in \mathcal{T}} \frac{H_{\lambda}}{H_{\lambda^{i-}}} \times\left(1-\frac{1}{x+\lambda_{i}-i}\right)=n-x+\frac{(x-n) g_{\lambda}(x+1)}{g_{\lambda}(x)} .
$$

Let us re-write (1.3)

$$
\sum_{\mu \in \lambda \backslash 1} \frac{H_{\lambda}}{H_{\mu}}=n .
$$

By subtracting (4.3) from (4.4) we obtain the following equivalent form of Theorem 1.1.

Theorem 4.1. We have

$$
\sum_{i \in \mathcal{T}} \frac{H_{\lambda}}{H_{\lambda^{i-}}} \times \frac{1}{x+\lambda_{i}-i}=x-\frac{(x-n) g_{\lambda}(x+1)}{g_{\lambda}(x)} .
$$

By the definitions of $\mathcal{T}$ and $\mathcal{B}$ we have

$$
\frac{(x-n) g_{\lambda}(x+1)}{g_{\lambda}(x)}=\frac{\prod_{i \in \mathcal{B}}\left(x+\lambda_{i}-i+1\right)}{\prod_{i \in \mathcal{T}}\left(x+\lambda_{i}-i\right)},
$$

so that Theorem 1.1 is also equivalent to the following result.

Theorem 4.2. We have

$$
\sum_{i \in \mathcal{T}} \frac{H_{\lambda}}{H_{\lambda^{i-}}} \times \frac{1}{x+\lambda_{i}-i}=x-\frac{\prod_{i \in \mathcal{B}}\left(x+\lambda_{i}-i+1\right)}{\prod_{i \in \mathcal{T}}\left(x+\lambda_{i}-i\right)} .
$$

For example, take $\lambda=55331$. Then $\mathcal{T}=2,4,5$ and $\mathcal{B}=1,3,5,6=$ $\{1,2+1,4+1,5+1\}$.

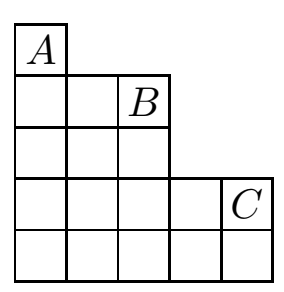

Fig. 4.1. in-corner

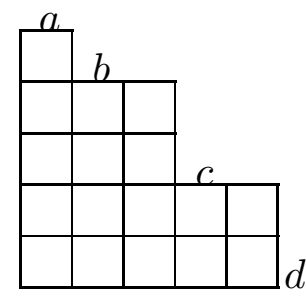

Fig. 4.2. out-corner

Hence $\lambda^{2-}=54331, \lambda^{4-}=55321$ and $\lambda^{5-}=55330$. Equality (4.7) becomes

$$
\begin{gathered}
\frac{H_{\lambda}}{H_{\lambda^{5}-}} \times \frac{1}{x-4}+\frac{H_{\lambda}}{H_{\lambda^{4}-}} \times \frac{1}{x-1}+\frac{H_{\lambda}}{H_{\lambda^{2-}}} \times \frac{1}{x+3} \\
=x-\frac{(x-5)(x-3)(x+1)(x+5)}{(x-4)(x-1)(x+3)} . \\
=\frac{17 x^{2}-38 x-75}{(x-4)(x-1)(x+3)} .
\end{gathered}
$$


Theorems 4.1 and 4.2 can be proved directly using the method used in the proof of Theorem 1.1. First, we must verify that the numerator in the right-hand side of (4.5) is a polynomial in $x$ whose degree is less than $(\leq)$ $\# \mathcal{T}-1$. By the partial fraction expansion technique it suffices to verify that (4.7) is true for all $x=i-\lambda_{i}(i \in \mathcal{T})$. This direct proof contains the main part of the proof of Theorem 1.1. However it does not make use of the fundamental relation (1.3) or (4.4). Thus, the following corollary of Theorem 4.2 makes sense.

Corollary 4.4. We have

$$
\sum_{\mu \in \lambda \backslash 1} \frac{H_{\lambda}}{H_{\mu}}=n
$$

Proof. Let $\# \mathcal{T}=k$. The right-hand side of (4.7) has the following form

$$
\frac{C x^{k-1}+\cdots}{x^{k}+\cdots} \text {. }
$$

We now evaluate the coefficient $C$. By (4.6) we can write $C=A-B$ with

$$
A=\left[x^{n-1}\right] x \prod_{i=1}^{n}\left(x+\lambda_{i}-i\right)=\sum_{1 \leq i<j \leq n}\left(\lambda_{i}-i\right)\left(\lambda_{j}-j\right)
$$

and

$$
\begin{aligned}
B & =\left[x^{n-1}\right](x-n) \prod_{i=1}^{n}\left(x+\lambda_{i}-i+1\right) \\
& =\sum_{1 \leq i<j \leq n}\left(\lambda_{i}-i+1\right)\left(\lambda_{j}-j+1\right)-n \sum_{1 \leq i \leq n}\left(\lambda_{i}-i+1\right) . \\
& =B_{1}-n \sum_{1 \leq i \leq n}\left(\lambda_{i}-i+1\right),
\end{aligned}
$$

where

$$
\begin{aligned}
B_{1} & =\sum_{1 \leq i<j \leq n}\left(\lambda_{i}-i+1\right)\left(\lambda_{j}-j+1\right) \\
& =\sum_{1 \leq i<j \leq n}\left(\left(\lambda_{i}-i\right)\left(\lambda_{j}-j\right)+\left(\lambda_{i}-i\right)+\left(\lambda_{j}-j\right)+1\right) \\
& =A+\sum_{1 \leq i<j \leq n}\left(\lambda_{i}-i\right)+\sum_{1 \leq i<j \leq n}\left(\lambda_{j}-j\right)+\left(\begin{array}{c}
n \\
2
\end{array}\right) \\
& =A+\sum_{1 \leq i \leq n}(n-i)\left(\lambda_{i}-i\right)+\sum_{1 \leq j \leq n}(j-1)\left(\lambda_{j}-j\right)+\left(\begin{array}{l}
n \\
2
\end{array}\right) \\
& =A+\sum_{1 \leq i \leq n}(n-1)\left(\lambda_{i}-i\right)+\left(\begin{array}{l}
n \\
2
\end{array}\right) .
\end{aligned}
$$




\section{Finally}

$$
\begin{aligned}
C & =A-B \\
& =-\sum_{1 \leq i \leq n}(n-1)\left(\lambda_{i}-i\right)-\left(\begin{array}{l}
n \\
2
\end{array}\right)+n \sum_{1 \leq i \leq n}\left(\lambda_{i}-i+1\right) \\
& =-\sum_{1 \leq i \leq n} n\left(\lambda_{i}-i\right)+\sum_{1 \leq i \leq n}\left(\lambda_{i}-i\right)-\left(\begin{array}{l}
n \\
2
\end{array}\right)+n \sum_{1 \leq i \leq n}\left(\lambda_{i}-i\right)+n^{2} \\
& =\sum_{1 \leq i \leq n}\left(\lambda_{i}-i\right)-\left(\begin{array}{l}
n \\
2
\end{array}\right)+n^{2} \\
& =n-\left(\begin{array}{c}
n+1 \\
2
\end{array}\right)-\left(\begin{array}{c}
n \\
2
\end{array}\right)+n^{2}=n .
\end{aligned}
$$

\section{References}

[AGJ88] Andrews, G; Goulden, I; Jackson, D. M., Generalizations of Cauchy's summation formula for Schur functions, Trans. Amer. Math. Soc., 310 (1988), pp. 805-820.

[FRT54] Frame, J. Sutherland; Robinson, Gilbert de Beauregard; Thrall, Robert M., The hook graphs of the symmetric groups, Canadian J. Math., 6 (1954), pp. 316-324.

[Ha08a] Han, Guo-Niu, Some conjectures and open problems about partition hook length, Experimental Mathematics, in press, 15 pages, 2008.

[Ha08b] Han, Guo-Niu, Discovering hook length formulas by expansion technique, in preparation, 42 pages, 2008.

[La01] Lascoux, Alain, Symmetric Functions and Combinatorial Operators on Polynomials, CBMS Regional Conference Series in Mathematics, Number 99, 2001.

[Ma95] Macdonald, Ian G., Symmetric Functions and Hall Polynomials, Second Edition, Clarendon Press, Oxford, 1995.

[St99] Stanley, Richard P., Enumerative Combinatorics, vol. 2, Cambridge University Press, 1999 .

[St08] Stanley, Richard P., Some combinatorial properties of hook lengths, contents, and parts of partitions, arXiv:0807.0383 [math.CO], 18 pages , 2008.

I.R.M.A. UMR 7501

Université Louis Pasteur et CNRS,

7, rue René-Descartes F-67084 Strasbourg, France guoniu@math.u-strasbg.fr 